\title{
Fringe tracking at the IOTA interferometer
}

Ettore Pedretti, Nathalie D. Thureau, Edward Wilson, Wesley Arthur Traub, John D. Monnier, et al.

Ettore Pedretti, Nathalie D. Thureau, Edward Wilson, Wesley Arthur Traub, John D. Monnier, Sam Ragland, Nathaniel P. Carleton, Rafael Millan-Gabet, F. Peter Schloerb, Michael K. Brewer, Jean-Philippe Berger, Marc G. Lacasse, "Fringe tracking at the IOTA interferometer," Proc. SPIE 5491, New Frontiers in Stellar Interferometry, (20 October 2004); doi: 10.1117/12.551625

Event: SPIE Astronomical Telescopes + Instrumentation, 2004, Glasgow, United Kingdom 


\title{
Fringe Tracking at the IOTA Interferometer
}

\author{
Ettore Pedretti ${ }^{a, b}$, Nathalie D. Thureau ${ }^{c \dagger}$, Edward Wilson ${ }^{d, e}$, Wesley A. Traub ${ }^{a}$, \\ John D. Monnier ${ }^{b}$, Sam Ragland ${ }^{a \dagger}$, Nathaniel P. Carleton ${ }^{a}$, Rafael Millan-Gabet ${ }^{f}$ \\ F. P. Schloerb ${ }^{g}$, M. K. Brewer ${ }^{g}$, J-P. Berger ${ }^{h}$ and Marc G. Lacasse ${ }^{a}$ \\ ${ }^{a}$ Smithsonian Astrophysical Observatory, 60, Garden Street, Cambridge, MA 02138, USA \\ ${ }^{b}$ University of Michigan Astronomy Dept., 500 Church Street, Ann Arbor, MI 48109, USA \\ ${ }^{c}$ Astrophysics Group, Cavendish Laboratory, Madingley Road, CB30HE, Cambridge, UK \\ ${ }^{d}$ Intellization, 454 Barkentine Ln, Redwood Shores, CA 94065-1126, USA \\ ${ }^{e}$ NASA Ames Research Center, MS 269-1, Moffett Field, CA 94035, USA

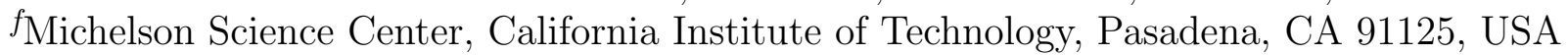 \\ ${ }^{g}$ University of Massachusetts, Amherst, MA 01003, USA \\ ${ }^{h}$ Laboratoire d' Astrophysique de Grenoble, BP 53 X, Grenoble Cedex 9, France
}

\begin{abstract}
We describe the fringe-packet tracking software installed at the infrared optical telescope array (IOTA). Three independently developed fringe-packet tracking algorithms can be used to equalise the optical path lengths at the interferometer. We compare the performance of these three algorithms and show results obtained tracking fringes for three independent baselines on the sky.
\end{abstract}

Keywords: infrared interferometry, fringe envelope tracking

\section{INTRODUCTION}

Observations performed with long-baseline ground-based optical/infrared interferometers are strongly affected by the turbulent atmosphere. Turbulence can reduce the visibility of fringes in many ways as described Porro ${ }^{1}$ for pupil-plane (or coaxial) beam combination and Thureau ${ }^{2}$ for image-plane beam combination. Turbulence randomly modulates the phases of the fringes which can then become unusable for image reconstruction. Using three or more telescopes allows getting rid of the atmospheric phase contamination. This is done through the closure-phase technique pioneered in radio astronomy ${ }^{3}$ and recently applied to long-baseline optical interferometry. ${ }^{4}$ The necessary condition for obtaining meaningful closure-phases is that the three fringe packets must happen in the same temporal interval. This is achieved by keeping the optical path difference (OPD) to a minimum.

There are two possible solutions for correcting the OPD in an optical or infrared interferometer affected by atmospheric and instrumental optical path errors: coherencing and cophasing. Coherencing relies on keeping the OPD between two telescopes within a fraction (about a wavelength) of the coherence envelope of the data (typically several to many wavelengths) and allows only short exposures to be taken. Cophasing intends to maintain the optical path within a fraction of a wavelength, allowing long exposures in the science beam; however cophasing suffers from fringe jumps since it cannot find the position of the white fringe itself but needs a coherencing algorithm to know the central position in a fringe envelope.

When IOTA relied on a single baseline the fringes were usually kept inside the scan interval manually by the observers. The installation of the third telescope at IOTA required an increase in the level of automation in the instrument, because manual tracking is not practical with three baselines to adjust. In particular the requirement to measure closure-phases necessitated a system capable of keeping the fringe packets in the centre of the scan using the existing hardware dedicated to acquiring science data. Fringes must be acquired in the same

${ }^{\dagger}$ Marie Curie Postdoctoral Fellow, ${ }^{\dagger}$ Michelson Postdoctoral Fellow

Further author information: (Send correspondence to E.P.)

E.P: E-mail: epedrett@umich.edu 

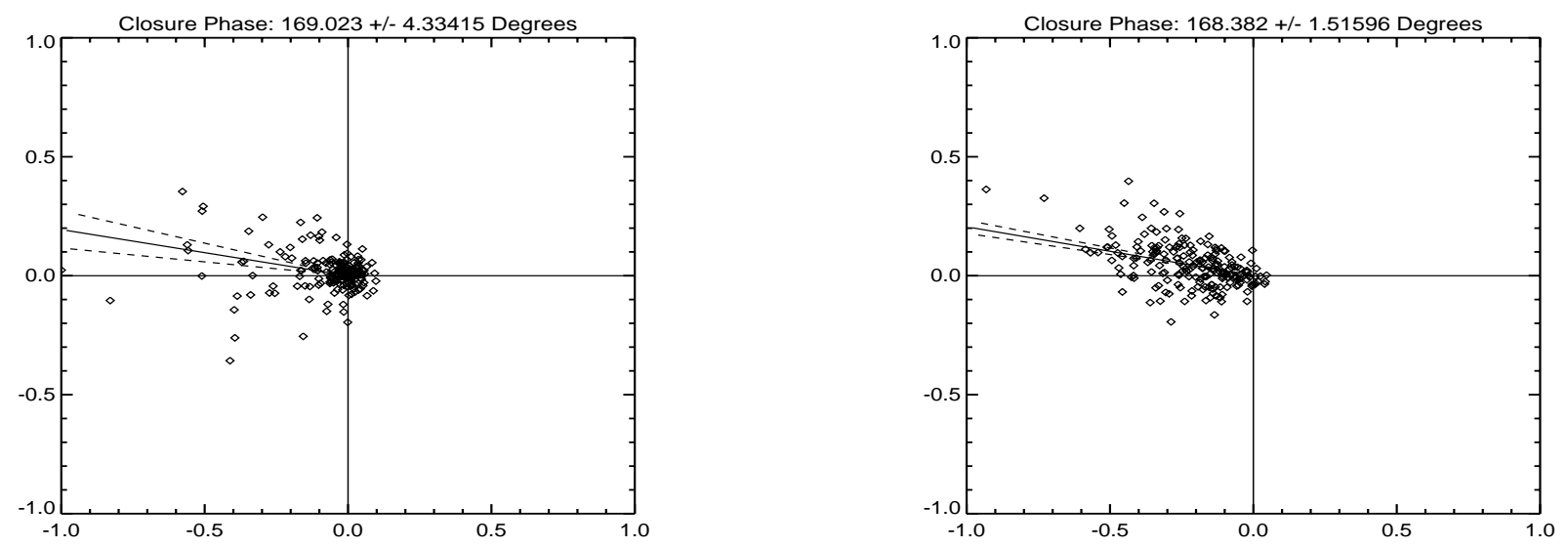

Figure 1. Closure phase measurement for the star WR140 $\left(m_{h}=5.3\right)$. The points represented on the plot are complex vectors normalised to unity. Each point is calculated using a single fringe measurement for each baseline (notably the $\mathrm{N}-\mathrm{S}, \mathrm{N}-\mathrm{W}$ and $\mathrm{W}-\mathrm{S}$ baselines, where the telescopes where positioned at $\mathrm{N}=35 \mathrm{~m}, \mathrm{~S}=15 \mathrm{~m}$ and $\mathrm{W}=10 \mathrm{~m}$ ). There are 200 points in the diagram. The vector represented in solid line is the average closure phase of the previous 200 vectors and the dashed line represents the error on the closure phase. The value of the mean closure phase and its associated error are also displayed at the top of the plot. Finally the left panel represents a closure phase measurement in open-loop mode (fringe tracker not active) while the right panel is the closed-loop case.

coherence time in order to measure a closure-phase. This is especially important at IOTA where the bandwidths are relatively large and the fringe packets quite narrow. Fig. 1 shows two measurements of closure phase. When the fringe tracker is switched off the closure phase is $169.0 \pm 4.3 \mathrm{deg}$ but it is only $168.4 \pm 1.5 \mathrm{deg}$ when the fringe tracker is operating (more than a factor 2 error reduction for the closed-loop case).

We present here three fringe-packet-tracking algorithms developed independently and tested at the IOTA interferometer. These algorithms do not require any additional hardware for their operation, but utilise the science data as fringe sensor and use the fringe-scanning piezo-mirrors and existing delay lines for fast path corrections.

\section{THE INSTRUMENT}

IOTA is a long-baseline optical interferometer located at the Smithsonian Institution Whipple Observatory on Mount Hopkins, AZ, comprising three $45-\mathrm{cm}$ diameter telescopes which can be positioned at 17 stations on an L-shaped track, where the arms are $15 \mathrm{~m}$ toward the south-east and $35 \mathrm{~m}$ toward the north-east. IOTA operated with 2 telescopes from $1995-2003$, and 3 telescopes since February 2002. The interferometer, ${ }^{5,6}$ has been used as a testbed for new cutting-edge technologies, ${ }^{7-9}$ and has produced recent science results in the 2 and 3-telescope configuration. ${ }^{10-18}$

The three beams arriving from the vacuum delay-line tank hit three dichroic mirrors which separate the visible and infrared light. The visible beam continues toward the star tracker servo system. The science infrared beam is reflected toward three flat mirrors and then three off-axis parabolas which focus the three beams on three single-mode (H-band) fibers feeding the IONIC-3T integrated-optics beam-combiner. ${ }^{19}$

Interference is achieved inside the integrated optics component, resulting in three output pairs $\pi$ radians out of phase in intensity. The interference fringes are recorded while two of the dichroics are piezo-driven to scan a path of about $100 \mu \mathrm{m}$ and $50 \mu \mathrm{m}$, respectively, in order to scan through the fringe packet in the three beams. The six combined beams are then focused on six separate pixels of the PICNIC array which serves as infrared science camera and fringe sensor for the fringe packet tracker. The path difference calculated by the packet-tracker is fed back to the piezo-scanning dichroics for a fast tracking response. The piezo scanners are off-loaded of their additional offsets every second, when a fraction of the error signal is sent to the short delay lines which are responsible for tracking the geometric delay caused by the rotation of the Earth. 


\section{CALCULATING THE FRINGE POSITION}

Three algorithms were independently developed and tested at IOTA. Since the installation of the third telescope, a new control system based on the VME-bus architecture and the VxWorks real-time operating system is operational. Three separate Motorola G3, 266-MHz CPUs control telescopes / delay lines, the visible star tracker, and data acquisition / fringe-tracking. This architecture simplifies enormously the task of synchronising in real-time functions which were previously implemented on separate personal-computers running different and non-real-time operating systems.

Previous attempts to implement a fringe packet tracker were not successful due to the large delay in communications among the different computers composing the control system ${ }^{20}$

\subsection{Tracking the Fringe Packet Using Double Fourier Interferometry}

This is a simple and fast algorithm ${ }^{21}$ (from now on algorithm "a"), also independently discovered by Tubbs, ${ }^{22}$ which uses double Fourier interferometry $(\mathrm{DFI})^{23}$ to extract wavelength dependent information from the fringe packet and calculate its group delay. This is done by scanning the fringe packet over an interval greater than the packet length, where the spectral resolution is proportional to the scan length. The group delay tracking (GDT) method has been applied to interferometry since the very beginning of the field, when Michelson ${ }^{24}$ used a prism for dispersing and acquiring fringes visually at the $20-\mathrm{ft}$ interferometer. Labeyrie ${ }^{25}$ used the same system and demonstrated fringe acquisition on a two-telescope interferometer.

Several system have been proposed since then, for correcting the optical path. ${ }^{26,27}$ GDT (also called dispersed fringe tracking when applied to image plane interferometry) has been routinely used at several interferometric facilities. ${ }^{28-30}$ In fact, at IOTA, GDT was selected as the original method of path difference monitoring in the visible, ${ }^{31-34}$ but the system was set aside in favor of making infrared observations, and therefore was never extensively used.

The discrete function $\bar{n}(j)$, is the average detector count (noiseless case).

$$
\bar{n}(j)=x_{t}\left\{1 \pm V \frac{\sin \left[\pi\left(j-J_{0}\right) \Delta m\right]}{\pi\left(j-J_{0}\right) \Delta m} \sin \left[\frac{2 \pi\left(j-J_{0}\right) m_{0}}{N}\right]\right\}
$$

where $j$ is the sample number, $m_{0}$ the discrete spatial frequency, $N$ is the number of samples, $x_{t}$ the total discrete intensity, $V$ the apparent visibility, $\Delta m$ the bandpass width and $J_{0}$ is the optical path that we want to calculate. We now estimate the Fourier transform $\widetilde{n}(m)$ of the recorded-fringes temporal scan and multiply $\widetilde{n}\left(m_{1}\right)$ by the complex conjugate of $\widetilde{n}\left(m_{2}\right)$ and define this as the cross-spectrum $X\left(m_{1}, m_{2}\right)$. This cross spectrum is also used in the Knox-Thompson algorithm ${ }^{35}$ for recovering near diffraction-limited images of stellar objects degraded by atmospheric seeing.

$$
\begin{aligned}
X\left(m_{1}, m_{2}\right) & =\left[\frac{i}{2} \exp \left(i \frac{2 \pi m_{1} J_{0}}{N}\right)\right] \cdot\left[\frac{i}{2} \exp \left(-i \frac{2 \pi m_{2} J_{0}}{N}\right)\right]^{*} \\
& =\frac{1}{4} \exp \left[-i 2 \pi J_{0}\left(m_{1}-m_{2}\right)\right]
\end{aligned}
$$

The cross-spectrum $X$ is now calculated for all the frequencies in the bandpass, in order to improve the signal-to-noise. The phases can in fact be averaged as vectors in the bandpass as shown by Buscher. ${ }^{36}$

$$
X=\sum_{m_{1}}\left\{\left[\frac{i}{2} \exp \left(i \frac{2 \pi m_{1} J_{0}}{N}\right)\right] \cdot\left[\frac{i}{2} \exp \left(-i \frac{2 \pi m_{2} J_{0}}{N}\right)\right]^{*}\right\}
$$

Here the sum is over all $m_{1}$ values within the bandpass, and $m_{2}=m_{1}+\Delta m_{12}$, and $\Delta m_{12}$ is a small integer. The phase noise is reduced by $\sqrt{N}$ if the noise is normally distributed, as it is the case for detector noise, which is dominant in near infrared.

$$
J_{0}=\frac{N}{2 \pi} \frac{\arg (\bar{X})}{\Delta m_{12}}
$$

Then $J_{0}$ can be calculated from Eqn. 4, here the returned value is expressed in sample number, starting from the origin. The frequency difference $\Delta m_{12}$ is usually kept to one sample, is $\Delta m_{12}=1$. 


\subsubsection{Algorithm summary}

1. The interference fringes are recorded while two of the dichroics are piezo-driven to scan a path of about $100 \mu \mathrm{m}$ and $50 \mu \mathrm{m}$, respectively. The time series $n d_{(j)}$ is recorded by the infrared science camera for the three beams.

2. The fast Fourier transform $X d_{(m)}$ of the time series $n d_{(j)}$ is computed separately for the three beams.

3. The cross-spectrum $X d$ is calculated for the 3 beams by multiplying $X d_{(m)}$ by its complex conjugated shifted by one sample.

4. The argument (phase) of the averaged complex numbers from the cross-spectrum $X d$ is computed in correspondence of the fringe peak in the cross-power-spectrum. The position of the fringe packets is obtained by multiplying the obtained argument by the length of the scan.

\subsection{Sliding Window Discrete Fourier Transform}

This algorithm, (from now on, algorithm "b") uses a totally different approach in order to detect the position of the fringe packet. A detailed description of the algorithm is given eleswhere. ${ }^{37-39}$ A single-frequency, slidingwindow discrete Fourier transform (DFT) is used to extract the packet envelope information from the fringe packet (Fig. 2.a-b).

The DFT works as a matched filter and returns only the fringe amplitude at a specific frequency, determined by the width of the sliding window. This operation is repeated for five different frequencies in order to take into account the jitter of the fringe frequency produced by the atmospheric piston (Fig. 2.c). The point-by-point maximum of the 5 smoothed DFT results is taken and convolved with a rectangular template. The maximum of the convolution (Fig 2.d-e) is the position of the fringe packet. Although the fringe frequency should be a known constant, in practice, it varies due to atmospheric distortion. This algorithm automatically adapts to account for fringe frequency changes between and within scans.

Once the position of the fringe packet has been found an estimation of the signal-to-noise must be passed to the baseline bootstrapping algorithm (see Sec 3.5), in order to know which fringe packet position information carries more "weight". Also, it is important to reject packet positions triggered by noise. This estimation is performed by comparing the value of the signal in the center of the packet with the value of the noise around the packet, as graphically shown in Fig. 2.f

\subsection{Description of the single-channel envelope detection algorithm}

We give in this section a description of the third algorithm (from now on algorithm "c") used to find the position of a fringe packet. For a more detailed description of the algorithm see Thureau. ${ }^{40}$ The algorithm takes a fringe template which is a sinusoid modulated by a roughly Gaussian envelope (see Fig. 3.a) and slides this template across the data, determining the correlation coefficient as a function of the template position.

The above operation corresponds to convolving the data with the template, which can be easily done in Fourier space. The data is fast Fourier transformed and multiplied by the fast Fourier transform of the template. Fourier transforming back to the time domain gives the correlation of the template with the data. We can at once convolve with two templates simultaneously, namely the envelope multiplied a cosine function and the envelope multiplied by a sine function, which corresponds to finding the real and imaginary parts of the fringe phasor. Multiplying a one-sided Fourier window function is indeed equivalent to convolving with a real cos function and an imaginary sine function. Taking the modulus squared of the resulting complex correlation therefore gives the total fringe power in these two templates as a function of time. The resulting function is a series of envelopes, which can be folded in time to give an average envelope, and this is what is plotted in Fig. 3.b.

Once the fringe envelopes have been computed, a routine based on a centroid calculation is applied, in order to determine the mean position of its centre. This mean position is then used as an estimate of the OPD. 


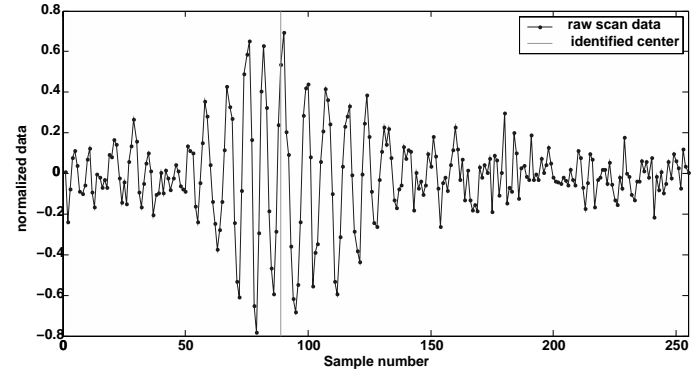

(c)

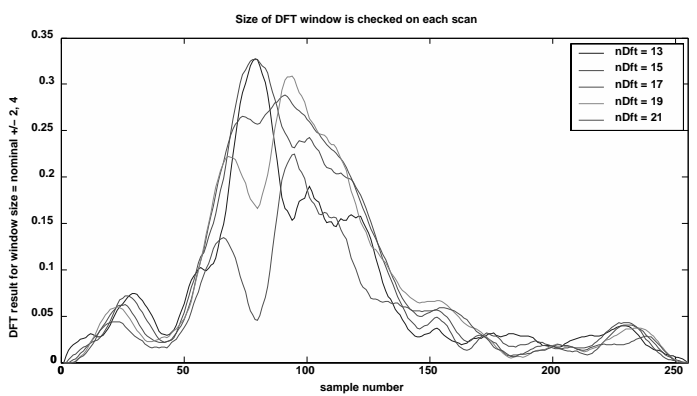

(e)

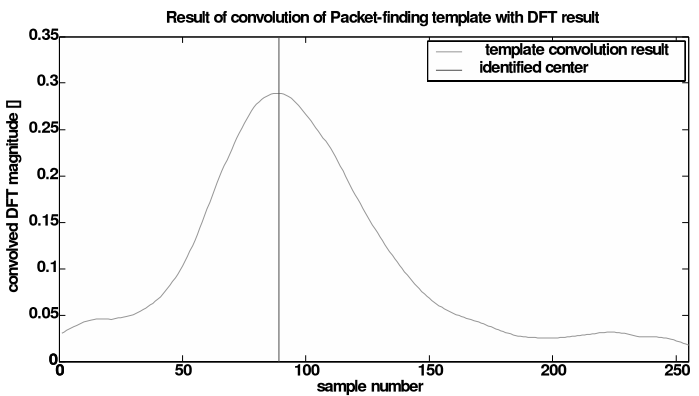

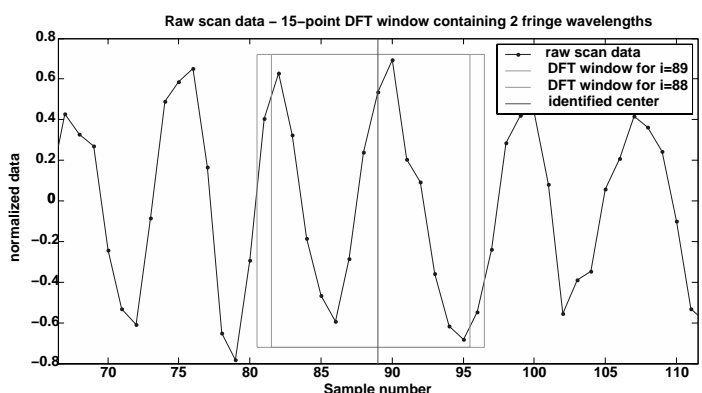

(d)

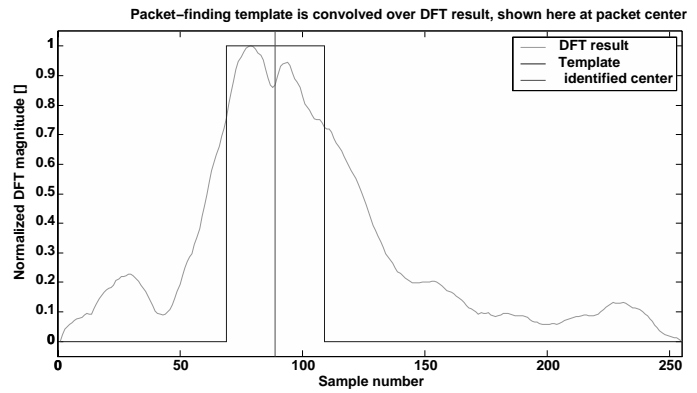

(f)

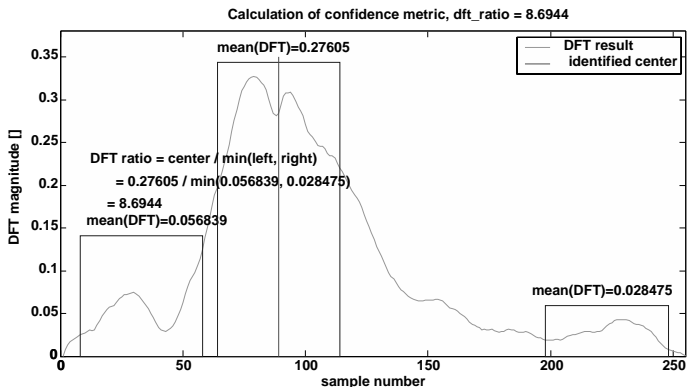

Figure 2. The raw scan with a high signal-to-noise fringe packet (a). Example of a window used to perform the sliding window DFT (b). DFT obtained for 5 different frequencies (c). Convolution of the DFT result with the packet finding window (d). Result of convolution and position of the fringe packet. Fringe packet signal-to-noise estimation (f). 

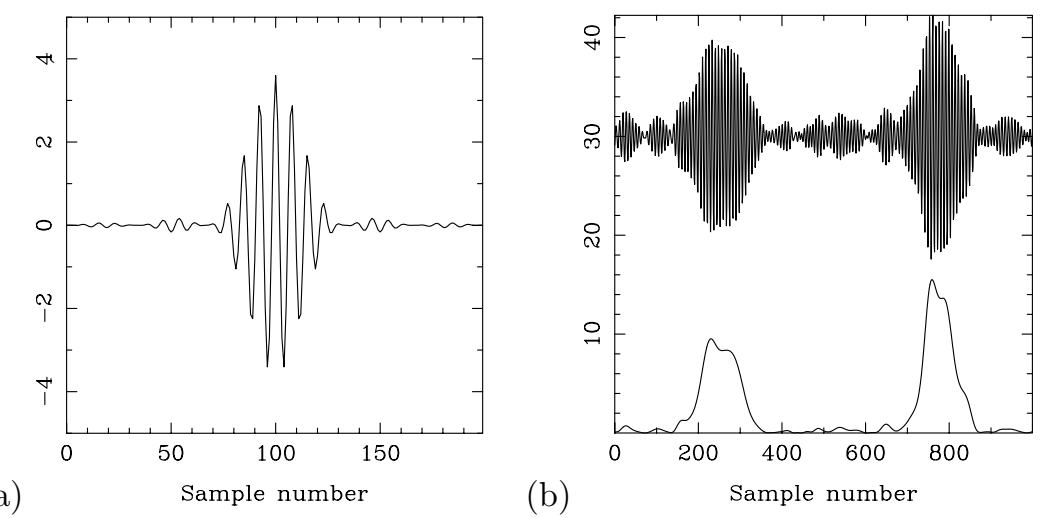

Figure 3. The template function (a). Example of intensity data and the resulting group-delay signal (b).

\subsection{Fringe Detection}

Since the first algorithm to be operational at IOTA was (a), depicted in Sec. 3.1 it was easier for the "guest" algorithms to use the existing routines developed for (a). This is the case for baseline bootstrapping (described in Sec. 3.5) and fringe detection (this is only true for algorithm (c), since algorithm (b) has its own fringe detection algorithm).

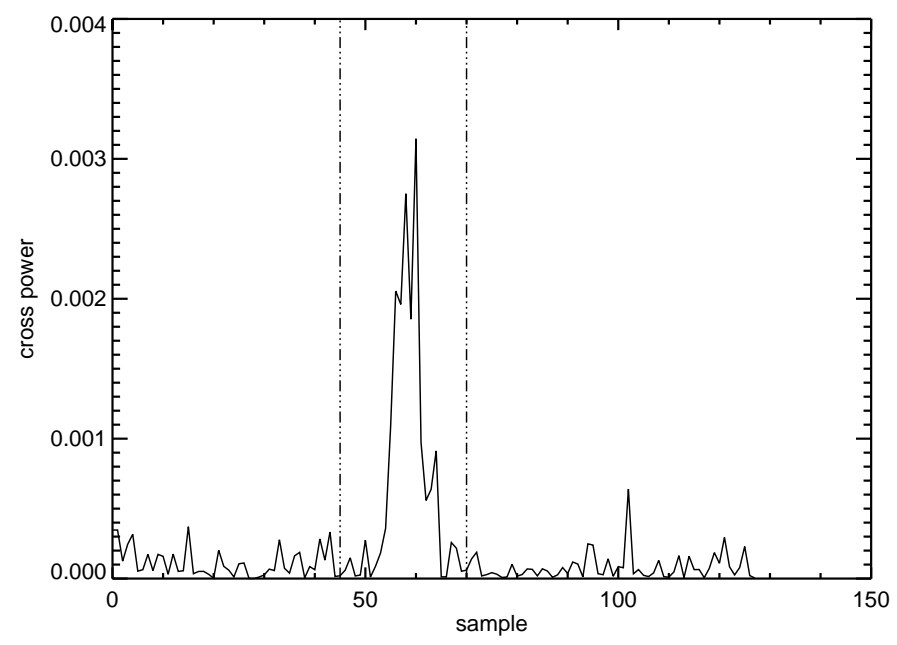

Figure 4. Signal-to-noise estimator for fringe detection. The power contained in the filter around the fringe peak is compared with the rest of the power outside the filter.

Fringe detection in (a) is obtained by comparing the signal-to-noise of the fringes with a fixed threshold entered as a parameter by the observer. The signal-to-noise is calculated from the cross spectrum $\left|X\left(f_{1}, f_{2}\right)\right|^{2}$, from Eqn. 3, dividing the averaged power inside the fringe peak by the averaged power outside the fringe peak.

\subsection{Baseline Bootstrapping}

With baseline bootstrapping we are capable of blind-tracking fringes on a baseline when the signal-to-noise of the fringes is too low, provided that good signal-to-noise is available on the other two baselines. For this reason, we calculate the optical path $J_{0}$ for three baselines even if we correct the path for two baselines. We can express one optical path as the weighted average of the other two optical paths, the weights being equal to the SNR for 
the fringes obtained on those baselines. The signal-to-noise is calculated from the cross spectrum $\left|X\left(f_{1}, f_{2}\right)\right|^{2}$, dividing the averaged power inside the fringe peak by the averaged power outside the fringe peak. We then observe that the optical path, in a closed loop must be equal to zero:

$$
J_{0} 1+J_{0} 2+J_{0} 3=0
$$

where $J_{0} 1$ and $J_{0} 2$ are the optical path where the servo loop is acting, while $J_{0} 3$ is the reference optical path. To the path $J_{0} 1, J_{0} 2$ and $J_{0} 3$ are associated the weights $w 1, w 2$ and $w 3$ respectively. We have two values for each optical path. One is the value obtained directly on that baseline (for example $J_{0} 1$ with weight $w 1$ ), the other is the value calculated from the linear combination of the other two baselines (for example $J_{0} 1^{\prime}=-J_{0} 2-J_{0} 3$ with weight $\left.w 1^{\prime}=(w 2 w 3) /(w 2+w 3)\right)$. The weighted average of $J_{0}$ and $J_{0}^{\prime}$ can then be written as: ${ }^{41}$

$$
\overline{J_{0} 1}=\frac{w 1 J_{0} 1+w 1^{\prime} J_{0} 1^{\prime}}{w 1+w 1^{\prime}}
$$

where $\overline{J_{0} 1}$ is the weighted-averaged path difference. Similarly for $\overline{J_{0} 2}$ :

$$
\begin{aligned}
\overline{J_{0} 2} & =\frac{w 2 J_{0} 2+w 2^{\prime} J_{0} 2^{\prime}}{w 2+w 2^{\prime}} \\
J_{0} 2^{\prime} & =-J_{0} 1-J_{0} 3 \\
w 2^{\prime} & =\frac{w 1 w 3}{w 1+w 3}
\end{aligned}
$$

The advantage of using a weighting system is that we do not have to select the best baseline values a priori, but rather the weighting allows them to be selected automatically.

\section{RESULTS AND PRELIMINARY COMPARISON}

The 3 algorithms perform equally well with moderately faint stars. Fig. 5 shows fringes recorded with algorithm (c) on the star $\alpha \mathrm{Vul}$, were the fringes are recorded with the fringe tracker switched on (top) and switched off

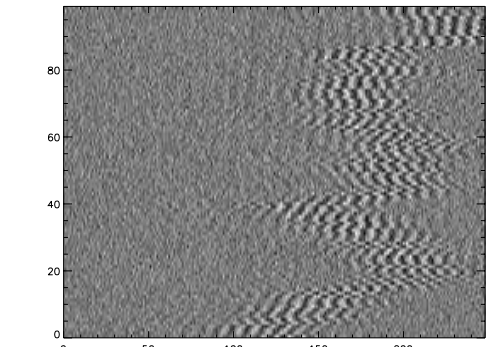

(a)

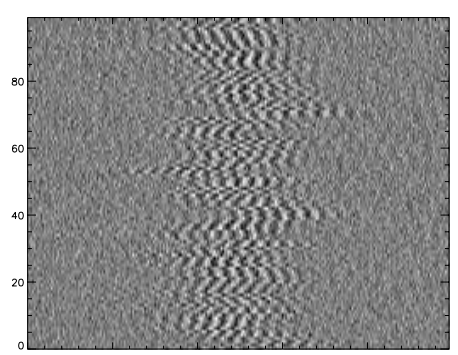

(c)

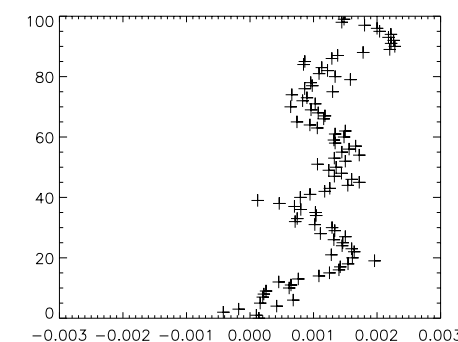

(b)

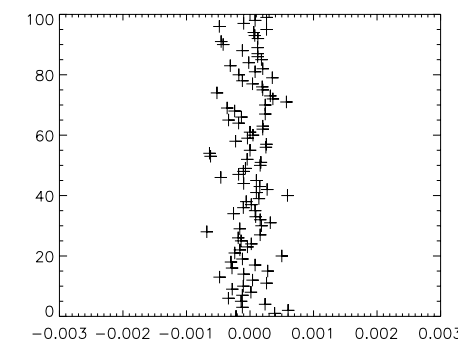

(d)

Figure 5. Fringes from the IOTA IONIC3T combiner on the PICNIC camera recorded in the H band on the star $\alpha$ Vul with a $5.5 \mathrm{~m}$ baseline. (a) Fringe pattern recorded without fringe tracking. (b) Fluctuations of the position of the fringe packet represented in (a). (c) Fringe pattern recorded while using algorithm (c). (d) Corresponding fringe packet position. 

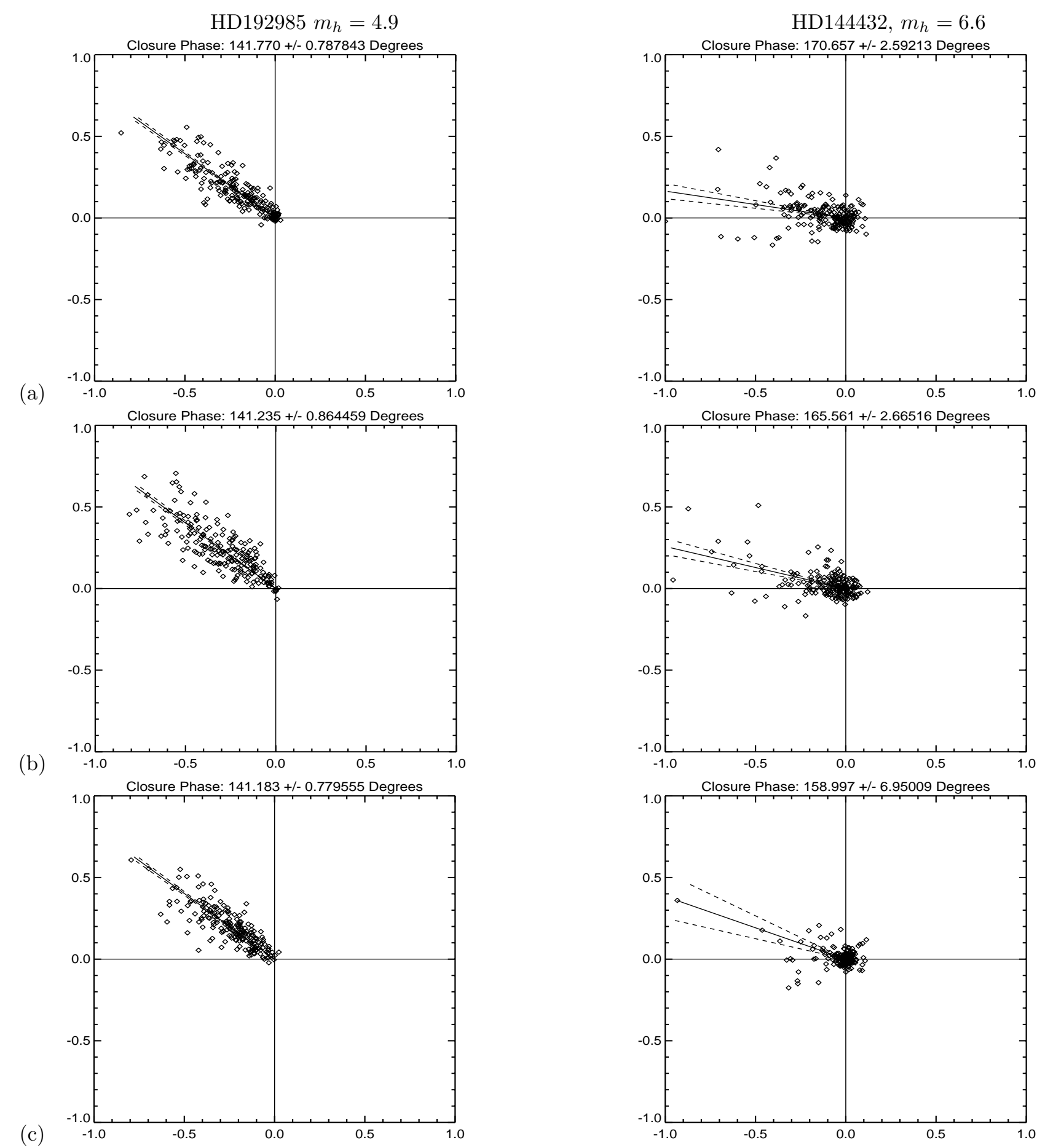

Figure 6. .Closure phase measured for the star HD192985 (left) and for the star HD144432 (right) during nights of bad seeing. Row (a) refers to the fringe packet tracking algorithm(a), row (b) to algorithm (b) and so forth. 
(bottom). The image shows the importance of using a fringe tracker to maintain coherence: the fringes would be lost very easily without actively tracking them.

The comparison of the algorithm is still work in progress. A crude performance comparison was done during the IOTA May 28 - June 07 observing run when time was available in between science targets. The algorithms were tested in identical conditions on target HD192985 $\left(m_{h}=4.9\right)$ the night of the 1st of June 2004 and on target HD144432 $\left(m_{h}=6.6\right)$ the night of the 6th of June 2004. The exposure were taken consecutively with the three algorithms. We use the closure-phase error as a rough estimator of the performance of the algorithms.

As stated before there is no tracking performance difference to be noticed at moderately low photon flux. Algorithm (b) is slower than the other two, but this does not compromise performance since the correction is applied to the piezo and to the delay lines after the scan and fly-back of the piezo. In fact the fringe packet is acquired, in servo mode, by scanning the optical path up to $100 \mu \mathrm{m}$ and the path correction is applied at the end of the scan. The total OPD has already changed quite a bit by the time the correction is applied ${ }^{21}$ and this introduces an error which is usually dominant.

At lower flux, we notice a moderate degradation of performance for algorithm (c) as it can be noticed in Fig. 6 , right-bottom pane. This algorithm has in fact the largest error on the closure phase. Since its implementation at the IOTA interferometer, algorithm (c) has been modified. The calculation of the fringe envelope position has been simplified and is now based on a cyclical centroid calculation. The new algorithm is currently being tested at COAST and it is expected to solve the problems encountered at low light levels.

\section{ACKNOWLEDGMENTS}

This research was made possible thanks to a Smithsonian Predoctoral Fellowship awarded to E. Pedretti, a Michelson Postdoctoral Fellowship awarded to S. Ragland and E Pedretti and a Marie Curie Fellowship (within the Fifth Framework programme "Improving Human Research Potential and the Socio-economic Knowledge Base) granted to N. D. Thureau (contract HPMF-CT-200-00966). The algorithm development work presented by E. Wilson was funded through Directors Discretionary Fund awards at NASA Ames Research Center.

\section{REFERENCES}

1. I. L. Porro, W. A. Traub, and N. P. Carleton, "Effect of Telescope Alignment on a Stellar Interferometer," Appl. Opt. 38, pp. 6055-6067, Oct. 1999.

2. N. D. Thureau, Contribution à l'interféromètrie optique à longue base en mode multi-tavelures. PhD thesis, Université de Nice-Sophia Antipolis - Faculté des sciences, 2001.

3. R. C. Jennison, "A phase sensitive interferometer technique for the measurement of the Fourier transforms of spatial brightness distributions of small angular extent," MNRAS 118, pp. 276--, 1958.

4. J. E. Baldwin, M. G. Beckett, R. C. Boysen, D. Burns, D. F. Buscher, G. C. Cox, C. A. Haniff, C. D. Mackay, N. S. Nightingale, J. Rogers, P. A. G. Scheuer, T. R. Scott, P. G. Tuthill, P. J. Warner, D. M. A. Wilson, and R. W. Wilson, "The first images from an optical aperture synthesis array: mapping of Capella with COAST at two epochs.," A\&A 306, pp. L13+, Feb. 1996.

5. W. A. Traub, N. P. Carleton, J. D. Bregman, M. K. Brewer, M. G. Lacasse, P. Maymounkov, R. MillanGabet, J. D. Monnier, S. Morel, C. D. Papaliolios, M. R. Pearlman, I. L. Porro, F. P. Schloerb, and K. Souccar, "Third telescope project at the IOTA interferometer," in Proc. SPIE, Interferometry in Optical Astronomy, Pierre J. Lena; Andreas Quirrenbach; Eds., 4006, pp. 715-722, July 2000.

6. W. A. Traub, A. Ahearn, N. P. Carleton, J. Berger, M. K. Brewer, K. Hofmann, P. Y. Kern, M. G. Lacasse, F. Malbet, R. Millan-Gabet, J. D. Monnier, K. Ohnaka, E. Pedretti, S. Ragland, F. P. Schloerb, K. Souccar, and G. Weigelt, "New Beam-Combination Techniques at IOTA," in Interferometry for Optical Astronomy II. Edited by Wesley A. Traub. Proceedings of the SPIE, Volume 4838, pp. 45-52 (2003)., pp. 45-52, Feb. 2003.

7. J. P. Berger, P. Haguenauer, P. Kern, K. Perraut, F. Malbet, I. Schanen, M. Severi, R. Millan-Gabet, and W. Traub, "Integrated optics for astronomical interferometry. IV. First measurements of stars," A\&A 376, pp. L31-L34, Sept. 2001. 
8. J. D. Monnier, J. P. Berger, R. Millan-Gabet, W. A. Traub, N. P. Carleton, E. Pedretti, C. M. Coldwell, and C. D. Papaliolios, "SMART Precision Interferometry at $794 \mathrm{~nm}$," in Proc. SPIE, Interferometry for Optical Astronomy, Wesley A. Traub, editor, 4838, pp. 1127-1138, Aug. 2003.

9. E. Pedretti, R. Millan-Gabet, J. D. Monnier, W. A. Traub, N. P. Carleton, J.-P. Berger, M. G. Lacasse, F. P. Schloerb, and M. K. Brewer, "The PICNIC Interferometry Camera at IOTA," PASP 116, pp. 377-389, Apr. 2004.

10. P. Kervella, V. Coudé du Foresto, G. Perrin, M. Schöller, W. A. Traub, and M. G. Lacasse, "The angular diameter and distance of the Cepheid $\zeta$ Geminorum," A\&A 367, pp. 876-883, Mar. 2001.

11. B. Mennesson, G. Perrin, G. Chagnon, V. C. d. Foresto, S. Ridgway, A. Merand, P. Salome, P. Borde, W. Cotton, S. Morel, P. Kervella, W. Traub, and M. Lacasse, "Evidence for Very Extended Gaseous Layers around O-rich Mira Variables and M Giants," ApJ 579, pp. 446-454, Nov. 2002.

12. G. Chagnon, B. Mennesson, G. Perrin, V. Coudé du Foresto, P. Salomé, P. Bordé, M. Lacasse, and W. Traub, "L'-Band Interferometric Observations of Evolved Stars," AJ 124, pp. 2821-2832, Nov. 2002.

13. K.-H. Hofmann, U. Beckmann, T. Blöcker, V. Coudé du Foresto, M. Lacasse, B. Mennesson, R. MillanGabet, S. Morel, G. Perrin, B. Pras, C. Ruilier, D. Schertl, M. Schöller, M. Scholz, V. Shenavrin, W. Traub, G. Weigelt, M. Wittkowski, and B. Yudin, "Observations of Mira stars with the IOTA/FLUOR interferometer and comparison with Mira star models," New Astronomy 7, pp. 9-20, Jan. 2002.

14. J. D. Monnier, R. Millan-Gabet, P. G. Tuthill, W. A. Traub, N. P. Carleton, V. Coude du Foresto, W. C. Danchi, M. G. Lacasse, S. Morel, G. Perrin, and I. Porro, "Aperture synthesis using multiple facilities: Keck aperture masking and the IOTA interferometer," in Proc. SPIE, Interferometry for Optical Astronomy, Wesley A. Traub, editor, 4838, pp. 379-386, Aug. 2003.

15. K. Ohnaka, U. Beckman, J. P. Berger, M. K. Brewer, K. Hofmann, M. G. Lacasse, V. Malanushenko, R. Millan-Gabet, E. Pedretti, J. D. Monnier, D. Schertl, F. P. Schloerb, V. Shenavrin, and W. A. Traub, "IOTA observation of the circumstellar envelope of R CrB," in Proc. SPIE, Interferometry for Optical Astronomy, Wesley A. Traub, editor, 4838, pp. 1068-1071, Aug. 2003.

16. R. Millan-Gabet, E. Pedretti, J. D. Monnier, W. A. Traub, F. P. Schloerb, N. P. Carleton, S. Ragland, M. G. Lacasse, W. C. Danchi, P. Tuthill, G. Perrin, and V. Coudé du Foresto, "Recent Science Results from the Two-Telescope IOTA," in Proc. SPIE, Interferometry for Optical Astronomy, Wesley A. Traub, editor, 4838, pp. 202-209, Aug. 2003.

17. G. Weigelt, U. Beckman, J. P. Berger, T. Bloecker, M. K. Brewer, K. Hofmann, M. G. Lacasse, V. Malanushenko, R. Millan-Gabet, J. D. Monnier, K. Ohnaka, E. Pedretti, D. Schertl, F. p. Schloerb, M. Scholz, W. A. Traub, and B. Yudin, "JHK-band spectro-photometry of T Cep with the IOTA interferometer," in Proc. SPIE, Interferometry for Optical Astronomy, Wesley A. Traub, editor, 4838, pp. 181-184, Aug. 2003.

18. J. D. Monnier, W. A. Traub, F. P. Schloerb, R. Millan-Gabet, J.-P. Berger, E. Pedretti, N. P. Carleton, S. Kraus, M. G. Lacasse, M. Brewer, S. Ragland, A. Ahearn, C. Coldwell, P. Haguenauer, P. Kern, P. Labeye, L. Lagny, F. Malbet, D. Malin, P. Maymounkov, S. Morel, C. Papaliolios, K. Perraut, M. Pearlman, I. L. Porro, I. Schanen, K. Souccar, G. Torres, and G. Wallace, "First Results with the IOTA3 Imaging Interferometer: The Spectroscopic Binaries $\lambda$ Virginis and WR 140," ApJ 602, pp. L57-L60, Feb. 2004.

19. J. Berger, P. Haguenauer, P. Y. Kern, K. Rousselet-Perraut, F. Malbet, S. Gluck, L. Lagny, I. SchanenDuport, E. Laurent, A. Delboulbe, E. Tatulli, W. A. Traub, N. Carleton, R. Millan-Gabet, J. D. Monnier, E. Pedretti, and S. Ragland, "An integrated-optics 3-way beam combiner for IOTA," in Interferometry for Optical Astronomy II. Edited by Wesley A. Traub . Proceedings of the SPIE, Volume 4838, pp. 1099-1106 (2003)., pp. 1099-1106, Feb. 2003.

20. S. Morel, W. A. Traub, J. D. Bregman, R. W. Mah, and E. Wilson, "Fringe-tracking experiments at the IOTA interferometer," in Proc. SPIE Vol. 4006, Interferometry in Optical Astronomy, Pierre J. Lena; Andreas Quirrenbach; Eds., pp. 506-513, July 2000.

21. E. Pedretti, Systèmes d' Imagerie Interferometrique. PhD thesis, Université de Provence (Aix-Marseille 1), 2003.

22. R. N. Tubbs, "Tracking and Characterising Atmospheric Phase Fluctuations at COAST." Part III undergraduate project report, May 1998, Cavendish Laboratory, Cambridge University, May 1998. 
23. J.-M. Mariotti and S. T. Ridgway, "Double Fourier spatio-spectral interferometry - Combining high spectral and high spatial resolution in the near infrared," $A \& A S$ 195, pp. 350-363, Apr. 1988.

24. A. A. Michelson and F. G. Pease, "Measurement of the diameter of alpha Orionis with the interferometer.," ApJ 53, pp. 249-259, May 1921.

25. A. Labeyrie, "Interference fringes obtained on VEGA with two optical telescopes," ApJ 196, pp. L71-L75, Mar. 1975.

26. F. Vakili and L. Koechlin, "Aperture synthesis in space - Computer fringe blocking," in New technologies for astronomy; Proceedings of the Meeting, Paris, France, Apr. 25, 26, 1989 (A90-37976 16-89). Bellingham, WA, Society of Photo-Optical Instrumentation Engineers, 1989, p. 109-116., pp. 109-116, Sept. 1989.

27. Y. Rabbia, S. Menardi, J. Gay, P. M. Bourlon, P. Antonelli, M. Dugue, J. Marchal, F. Reynaud, M. Faucherre, and N. Hubin, "Prototype for the European Southern Observatory VLTI fringe sensor," in Proc. SPIE Vol. 2200, p. 204-215, Amplitude and Intensity Spatial Interferometry II, James B. Breckinridge; Ed., pp. 204-215, June 1994.

28. S. Robbe, B. Sorrente, F. Cassaing, Y. Rabbia, G. Rousset, L. Dame, P. Cruzalebes, and G. Schumacher, "Active phase stabilization at the I2T: implementation of the ASSI table," in Proc. SPIE Vol. 2200, p. 222-230, Amplitude and Intensity Spatial Interferometry II, James B. Breckinridge; Ed., pp. 222-230, June 1994.

29. P. R. Lawson, "Group-delay tracking in optical stellar interferometry with the fast Fourier transform," Optical Society of America Journal 12, pp. 366-374, Feb. 1995.

30. L. Koechlin, P. R. Lawson, D. Mourard, A. Blazit, D. Bonneau, F. Morand, P. Stee, I. Tallon-Bosc, and F. Vakili, "Dispersed fringe tracking with the multi- $r_{o}$ apertures of the Grand Interferometre a 2 Telescopes," Appl. Opt. 35, pp. 3002-3009, June 1996.

31. P. Nisenson and W. Traub, "Magnitude Limit of the Group Delay Fringe Tracking Method for Long Baseline Interferometry," in Interferometric Imaging in Astronomy, pp. 129-+, 1987.

32. W. A. Traub and M. G. Lacasse, "Laboratory Measurements of Visibility Using Dispersed Fringes in Wavenumber Space," in NOAO-ESO Conference on High-Resolution Imaging by Interferometry: GroundBased Interferometry at Visible and Infrared Wavelengths, Garching bei München, Germany, Mar. 15-18, 1988. Edited by F. Merkle, ESO Conference and Workshop Oroceedings No. 29, p.947, 1988, pp. 947-+, 1988.

33. W. A. Traub, "Constant-dispersion grism spectrometer for channeled spectra," Optical Society of America Journal 7, pp. 1779-1791, Sept. 1990.

34. W. A. Traub, M. G. Lacasse, and N. P. Carleton, "Spectral dispersion and fringe detection in IOTA," in Amplitude and intensity spatial interferometry; Proceedings of the Meeting, Tucson, AZ, Feb. 14-16, 1990 (A91-30676 12-89). Bellingham, WA, Society of Photo-Optical Instrumentation Engineers, 1990, $p$. 145-152. Research supported by NASA and Smithsonian Institution., pp. 145-152, Aug. 1990.

35. K. T. Knox and B. J. Thompson, "Recovery of images from atmospherically degraded short-exposure photographs," ApJ 193, pp. L45-L48, Oct. 1974.

36. D. F. Buscher Ph.D. Thesis, 1988.

37. E. Wilson and R. W. Mah, "Online fringe tracking and prediction at IOTA," in Proc. SPIE Vol. 3749, 18th Congress of the International Commission for Optics, Alexander J. Glass; Joseph W. Goodman; Milton Chang; Arthur H. Guenther; Toshimitsu Asakura; Eds., pp. 691-692, July 1999.

38. E. Wilson, E. Pedretti, J. D. Bregman, R. Mah, and W. A. Traub, "Adaptive DFT-based fringe tracking and prediction at IOTA," in Proc. SPIE, New Frontiers is Stellar Interferometry, Wesley A. Traub; Eds., 5491, June 2004.

39. E. Wilson, E. Pedretti, J. D. Bregman, R. Mah, and W. A. Traub, "Adaptive DFT-based interferometer fringe tracking," EURASIP Journal on Applied Signal Processing, 2005.

40. N. D. Thureau, R. C. Boysen, D. F. Buscher, C. A. Haniff, E. Pedretti, P. J. Warner, and J. S. Young, "Fringe envelope tracking at COAST," in Interferometry for Optical Astronomy II. Edited by Wesley A. Traub . Proceedings of the SPIE, Volume 4838, pp. 956-963 (2003)., pp. 956-963, Feb. 2003.

41. P. R. Bevington and D. K. Robinson, Data reduction and error analysis for the physical sciences, New York: McGraw-Hill, —c1992, 2nd ed., 1992. 